\title{
A Comparative Study of Three Different Chemical Vapor Deposition Techniques of Carbon Nanotube Growth on Diamond Films
}

\author{
Betty T. Quinton, ${ }^{1,2}$ Paul N. Barnes, ${ }^{3}$ Chakrapani V. Varanasi, ${ }^{4}$ Jack Burke, ${ }^{5}$ \\ Bang-Hung Tsao, ${ }^{5}$ Kevin J. Yost, ${ }^{1}$ and Sharmila M. Mukhopadhyay ${ }^{2}$ \\ ${ }^{1}$ Air Force Research Laboratory (AFRL), Wright-Patterson, Air Force Base, OH 45433, USA \\ ${ }^{2}$ Wright State University, Dayton, OH 45420, USA \\ ${ }^{3}$ Army Research Laboratory, Adelphi, MD 20783, USA \\ ${ }^{4}$ Army Research Office, Research Triangle Park, NC 27709, USA \\ ${ }^{5}$ University of Dayton Research Institute (UDRI), Dayton, OH 45469, USA
}

Correspondence should be addressed to Betty T. Quinton; bettyyanguic@gmail.com

Received 28 November 2012; Accepted 17 February 2013

Academic Editor: Lijun Ji

Copyright (C) 2013 Betty T. Quinton et al. This is an open access article distributed under the Creative Commons Attribution License, which permits unrestricted use, distribution, and reproduction in any medium, provided the original work is properly cited.

\begin{abstract}
This paper compares between the methods of growing carbon nanotubes (CNTs) on diamond substrates and evaluates the quality of the CNTs and the interfacial strength. One potential application for these materials is a heat sink/spreader for high-power electronic devices. The CNTs and diamond substrates have a significantly higher specific thermal conductivity than traditional heat sink/spreader materials making them good replacement candidates. Only limited research has been performed on these CNT/diamond structures and their suitability of different growth methods. This study investigates three potential chemical vapor deposition (CVD) techniques for growing CNTs on diamond: thermal CVD (T-CVD), microwave plasma-enhanced CVD (MPECVD), and floating catalyst thermal CVD (FCT-CVD). Scanning electron microscopy (SEM) and high-resolution transmission electron microscopy (TEM) were used to analyze the morphology and topology of the CNTs. Raman spectroscopy was used to assess the quality of the CNTs by determining the $I_{D} / I_{G}$ peak intensity ratios. Additionally, the CNT/diamond samples were sonicated for qualitative comparisons of the durability of the CNT forests. T-CVD provided the largest diameter tubes, with catalysts residing mainly at the CNT/diamond interface. The MPE-CVD process yielded non uniform defective CNTs, and FCT-CVD resulted in the smallest diameter CNTs with catalyst particles imbedded throughout the length of the nanotubes.
\end{abstract}

\section{Introduction}

Carbon nanotubes (CNTs) are lightweight materials that express superior mechanical, electrical, and thermal properties [1-4]. Diamond films are well known for their hardness and scratch resistance combined with excellent thermal conductivity $[5,6]$. Successful CNT growth on a diamond substrate creates a unique all-carbon structure that can be beneficial for advanced power and electronic applications. Various methods such as chemical vapor deposition (CVD), laser ablation, thermal evaporation, arc discharge, and glow discharge have been used to grow CNTs on different surfaces [7-9] including diamond substrates and nanoparticles [9-11].
Among these methods, CVD is perhaps the most promising and scalable approach for future power and electronic devices.

There are many different varieties of CVD used to grow CNTs. The variations depend on power sources, type of catalyst deposition, gas composition, and operating temperatures. In addition to variations of the CVD process, CNT growth is expected to depend significantly on the chemistry, morphology, and activity of the substrate. Catalyst and substrate interactions can create differences in root growth or tip growth mechanisms, size distribution, and defects in CNTs growth [12-14]. 
Several CVD approaches have been investigated and documented on common electronic substrates such as silicon, but no work seems to have been reported on specialty substrates such as CVD diamond. It is desirable to test the applicability of these techniques on diamond substrates and compare the CNTs obtained from different CVD growth techniques. This would enable future device manufacturers to select the most appropriate technique for a specific application. This study compares three types of CVD techniques believed to be the most probable candidate techniques to be scaled up and utilized for future power devices. They all utilize transition metal catalysts that can readily form metastable carbides needed for $\mathrm{CNT}$ growth on any substrate [15-17]. The three CVD approaches selected were thermal CVD (T-CVD) with presputtered metal catalyst, microwave plasma-enhanced CVD (MPE-CVD) with presputtered metal catalyst, and floating catalyst thermal CVD (FCT-CVD) with xylene and ferrocene liquid mixture without any prior catalyst deposition. T-CVD is a low-cost system that can easily be set up to grow CNTs. In comparison to arc discharge, T-CVD operates at a lower temperature which increases the range of substrate material selections. However, the resulting CNTs' structure may be defective compared to arc discharge or laser ablation [18]. MPE-CVD is very suitable for large surface CNT production and can grow CNTs at even lower temperatures than T-CVD [19]. Both these techniques involve predeposition of catalyst on the substrate. The FCT-CVD introduces the carbon and catalyst simultaneously on the substrate via a gas mixture. This approach cuts out the first step of catalyst deposition giving it a potential economic advantage for future scale-up. However, it coats everything in the growth chamber and may be problematic when selective area growth is required [20].

The CNT forests grown have been investigated in detail, and each sample has been analyzed using a scanning electron microscope (SEM), transmission electron microscope (TEM), Raman spectroscopy, and energy dispersive X-ray spectroscopy (EDS) to fully characterize the structures.

\section{Experimental Methods}

2.1. Sample Preparation. Polished free-standing diamond substrates grown by a CVD technique were purchased from $\mathrm{SP}^{3}$ Diamond Technologies Inc. The substrate measurements were $5 \times 5 \mathrm{~mm}^{2}$, with a $400 \mu \mathrm{m}$ thickness. The surface roughness was determined to be $5 \mathrm{~nm}$ as measured by an atomic force microscope. Three CVD-based CNT growth techniques have been investigated: (i) T-CVD, (ii) MPECVD, and (iii) FCT-CVD. The first two techniques involved predeposition of seed catalysts prior to CNT growth and were optimized first on an electronic grade silicon substrate with a layer of thermal silicon dioxide layer on the surface. The last one had the catalyst source combined with the carbon source in the feeder gas and had been optimized earlier on graphitic carbon substrates.

Due to limited quantities of CVD diamond substrates, each -of the growth methods described below were first tested and optimized using other standard substrates before deposition on the diamond. While it is recognized that optimization of parameters on identical diamond substrates would be ideal, the large number of diamond substrates necessary for such an undertaking was not available. It was, however, verified that the growth conditions on standard substrates were repeatable on other available substrates. As for growth parameters of CNTs on the diamond available in the literature the values reported for T-CVD and MPE-CVD were found to be very comparable to ones derived in house on standard substrates $[10,21]$. There is no reported growth on CNTs on diamond substrate using the FCT-CVD method. However, this technique involves precoating the substrate with a plasma-enhanced oxide layer, which is expected to make the CNT growth more independent of the underlying substrate. Additional optimization and refinement on specific diamond substrates may be performed in the future as more varieties of diamond substrates become available in larger quantities. At the current time, Raman spectroscopy was used as an indicator of CNT quality, and the $D$-peak to $G$-peak intensity ratios from CNTs obtained on all samples were more than satisfactory. Successful and repeatable experiments from earlier research indicated that the best CNT forests were obtained by using T-CVD and MPE-CVD growth processes with nickel and iron catalysts, respectively. For the FCT-CVD approach, ferrocene is used as an iron source to promote CNT growth. Based on these repeatable results, the CNT growth parameters were used to grow CNTs on the diamond films.

2.2. T-CVD Method. Polished free-standing CVD diamond substrates were cleaned in an ultrasonic acetone bath for 5 minutes followed by 5 minutes in an ultrasonic isopropanol bath. After cleaning, the samples were placed into a radio frequency (RF) sputtering system loaded with a $99.999 \%$ pure nickel target. The sputtering system uses RF power to create a plasma plume that deposits material from the target to the substrate surface. The substrate was first sputtered with a $10 \mathrm{~nm}$ thin film of nickel in a sputter chamber. Then, the nickel-sputtered sample then placed into a T-CVD furnace. The furnace was heated from room temperature to the growth temperature of $800^{\circ} \mathrm{C}$ in 20 minutes with an argon/5\% hydrogen $\left(\mathrm{Ar} / 5 \% \mathrm{H}_{2}\right)$ gas mixture at a flow rate of 300 standard cubic centimeters per minute $(\mathrm{sccm})$. Upon reaching the growth temperature, the sample was held for 10 minutes to allow the thin nickel film to break up into nanoscale islands. Then $400 \mathrm{sccm}$ of argon $/ 10 \%$ methane $\left(\mathrm{Ar} / 10 \% \mathrm{C}_{2} \mathrm{H}_{2}\right)$ was introduced as the carbon feedstock source at the end of the 10 -minute heat treatment. The growth process continued for 2 hours followed by a slow cool to room temperature again using a flow of $300 \mathrm{sccm}$ of a $\left(\mathrm{Ar} / 5 \% \mathrm{H}_{2}\right)$ gas mixture. The entire growth process was performed at a pressure of 90 torr.

2.3. MPE-CVD Method. The same sample cleaning procedures discussed above were followed here. The sputtering process was used again, but with a $99.999 \%$ pure iron target to form a $10 \mathrm{~nm}$ thin film of iron on the CVD diamond samples. The sample was loaded into the MPE-CVD chamber. The furnace was heated from room temperature to $400^{\circ} \mathrm{C}$ with pure hydrogen gas flowing at $150 \mathrm{sccm}$. Once the 
temperature reached $400^{\circ} \mathrm{C}$, a microwave frequency was used on the hydrogen gas to induce a hydrogen plasma within the chamber. The sample was held for 5 minutes with the hydrogen plasma at $400^{\circ} \mathrm{C}$ to allow iron island formation. After 5 minutes of annealing in plasma, the temperature was increased to the $650^{\circ} \mathrm{C}$ growth temperature, followed by the introduction of $15 \mathrm{sccm}$ of methane gas as a carbon source. Methane gas was allowed to flow for 5 minutes for growth to take place. After that, the methane gas was shut off. With hydrogen gas still flowing into the chamber, the current source for the plasma was also shut off. Finally, the heating unit was turned off to allow the system to cool naturally.

\subsection{FCT-CVD Method. The free-standing CVD diamond} samples were treated with microwave plasma containing dimethyl sulfoxide (DMSO) to deposit a thin film of silica, $100 \mathrm{~nm}$ in thickness, onto the CVD diamond substrate. Note that there is a correlation between the silica thickness and the quality of CNTs and that this silica layer is required in order for CNTs to grow using FCT-CVD methods [22, 23]. The FCT-CVD system is a two-stage furnace with Ar gas flowing at $600 \mathrm{sccm}$ and hydrogen gas flowing at $45 \mathrm{sccm}$. The low-temperature furnace is kept at $250^{\circ} \mathrm{C}$, while the hightemperature furnace is ramped up to the growth temperature of $750^{\circ} \mathrm{C}$. Ferrocene was dissolved into a xylene solvent in a 0.008 : 1 molar volume ratio. The xylene/ferrocene liquid mixture serves as a carbon feed stock as well as an iron catalytic particle source carrier. During growth, xylene/ferrocene was introduced at a flow rate of $3 \mathrm{~mL} / \mathrm{hr}$ via a syringe pump. The growth time was 20 minutes followed by a cooldown with $600 \mathrm{sccm}$ of Ar gas. Details of silica treatment as well as the CNT growth parameters can be found in earlier publications [24].

2.5. Characterization. The resulting samples were characterized using a FEI Sirion high-resolution SEM for analysis of carpet surface and nanotube morphology. Cross-sectional views of the interfaces are needed to understand how catalyst particles play a role during CNT growth. A thin TEM foil of the cross section of interest was made using a DB235 FEI focused ion beam. To protect CNTs from the gallium ion etching, a layer of platinum cap was sputtered on top of the CNT before the trenching process began for a lift out sample. The resulting foil was attached to a molybdenum TEM grid for final thinning until a transparent foil was formed. The TEM foil was first analyzed using a Philips CM200 TEM for general appearances and identified the different layers that it contains. The foils containing CNTs with both catalyst and substrate intact were then analyzed for elemental compositions by energy dispersive $\mathrm{X}$-ray spectroscopy (EDS) using the FEI Titan 80-300 TEM. In addition to the imaging analysis, Raman spectroscopy analysis was used to provide a qualitative value for the growth structures of CNTs. It uses the intensity ratio of the disorder peak ( $D$-peak) over the graphite peak (G-peak) to determine this qualitative value [25]. In order to monitor the durability and strength of bonding between CNTs and the diamond substrate, a sonication test was performed on samples made from each growth method.
This test uses Bransonic 3210, an ultrasonic cleaner with $40 \mathrm{kHz}$ frequency. The samples were sonicated for 15 minutes while submerged in deionized water. The results from the previous analyses are discussed in the next section.

\section{Results and Discussions}

3.1. Initial Catalyst Distribution in Preseeded Samples. It is well established that the underlying catalysts play a big role in the structure and properties of the resulting CNTs. The three different techniques compared here have two different approaches of introducing the catalyst: T-CVD and MPECVD involve predeposition of nickel and iron catalysts, respectively, while FCT-CVD technique introduces catalyst with the feeder gas during CNT growth. In the first two cases, the morphology of the starting catalyst film could be analyzed prior to CNT growth. For the last case, there is no precursor catalyst film, and catalyst morphology can only be seen after the CNT layer is formed, as discussed later.

Figure 1(a) shows that thermal treatment of the $10 \mathrm{~nm}$ nickel film covering the polished diamond surface resulted in nanoislands with average diameters of $53 \mathrm{~nm}$ distributed uniformly across the substrate surface. According to Homma et al. [26], the resulting particle size can be modified and is dependent on the film thickness. A thinner film thickness using the procedure mentioned above provided a low CNTs growth yield. Because of this, the study uses $10 \mathrm{~nm}$ film thicknesses as the testing film thickness.

Figure 1(b) shows the SEM surface analysis of a sample treated at $400^{\circ} \mathrm{C}$ with hydrogen plasma inside the MPECVD system for 5 minutes. The image shows that the iron catalyst agglomerated into a continuous uneven film rather than solidified as isolated nanoparticles.

3.2. Nanotube Morphology. Figure 2 shows SEM images of the CNT layer formed on diamond film using the three different techniques. Figure 2(a) is the image of T-CVD grown CNTs which have an average tube diameter of $61 \mathrm{~nm} \pm 12 \mathrm{~nm}$, with smooth and uniform tubular structure. This correlates well with the uniform size distribution of nickel islands of similar sizes seen in Figure 1(a).

Figure 2(b) is an SEM image of MPE-CVD-grown CNTs that indicates that the CNTs are not uniform and contain various defects as well as variation in diameters. This may be the result of the seed catalyst layer consisting of irregularshaped particles. The nonuniformity of individual tubes may also be influenced by defects developed during formation. The MPE-CVD method uses a hydrogen plasma that forms a reducing environment within the chamber. The SEM image taken can be compared to the study done by Behr which indicates that hydrogen can etch CNTs during the growth process. This can create surface defects throughout the growth process [27]. Some studies suggest that exposing growing CNTs to hydrogen-rich environments convert them into diamonds $[28,29]$. Figure 2(b) shows the MPE-CVD CNTs tubes with defects similar to the ones reported by Behr after exposure to the hydrogen-rich environment. 


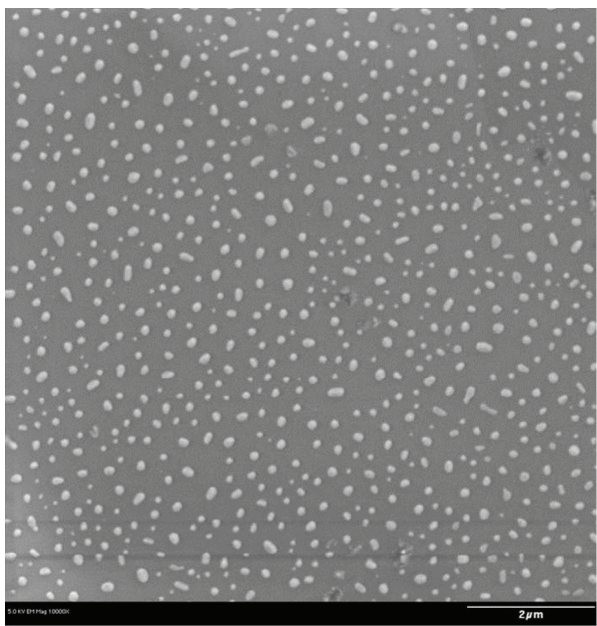

(a)

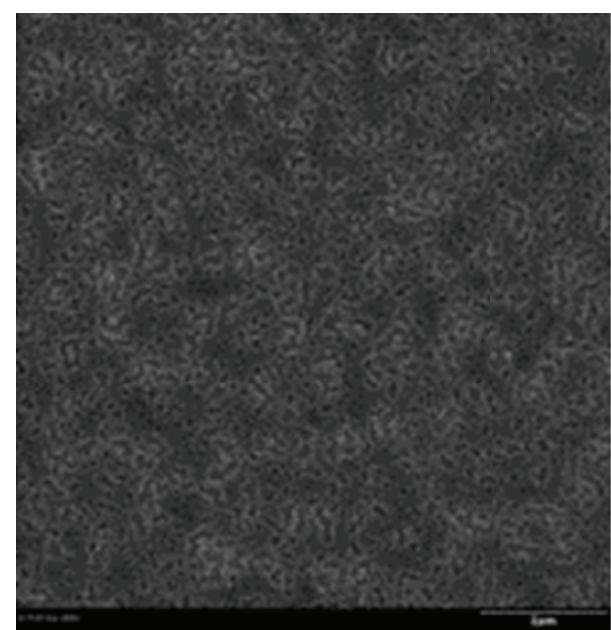

(b)

FIgURE 1: (a) Ni catalyst after thermal treatment in T-CVD. (b) Fe catalyst after thermal treatment in MPE-CVD with hydrogen plasma.

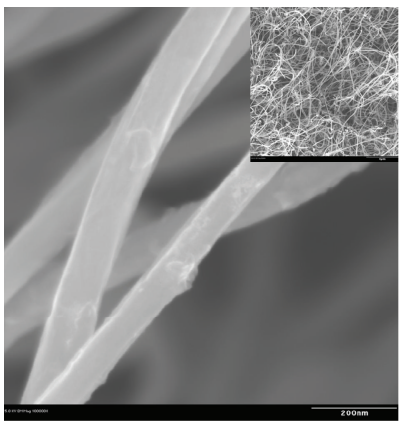

(a)

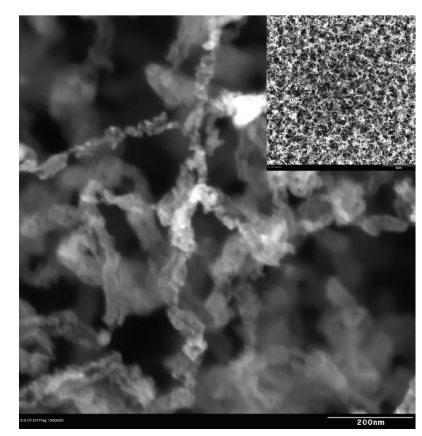

(b)

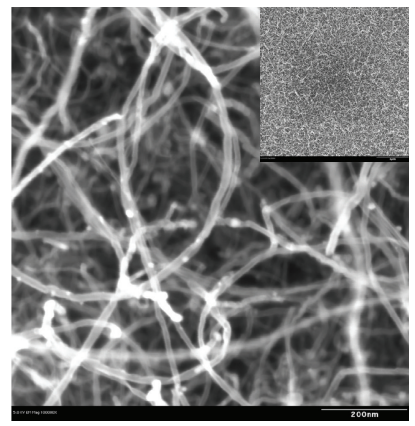

(c)

Figure 2: (a) T-CVD-grown CNTs. (b) MPE-CVD-grown CNTs. (c) FCT-CVD-grown CNTs.

Figure 2(c) shows an image of FCT-CVD-grown CNTs. This figure indicates that the CNTs are uniform in diameter and grow as densely entangled forests. These CNTs have average diameters of $12 \mathrm{~nm} \pm 1.8 \mathrm{~nm}$, making them the smallest of the three growth methods. The image also suggests that there are particles residing on the outer surface of the CNTs. This is expected because the xylene/ferrocene mixture provides continuous source of iron particles throughout the entire process, some of which are expected to attach to the growing CNT surface. The diameter of the outer particles can provide an estimate of iron catalyst size, and they have an averaging diameter size of $12 \mathrm{~nm} \pm 2.2 \mathrm{~nm}$.

\subsection{Catalyst Distribution in the Final CNT-Covered Diamond} Structure. Figures 3-5 show high-resolution TEM images with EDS elemental mapping. Figure 3 indicates that in TCVD, nickel particles are encapsulated with a layer of carbon, and CNT growth originates from the outer carbon layer. This explains why the average CNT diameter is slightly higher than the average nickel catalyst diameter as shown later. The encapsulated catalyst particle remains anchored at the diamond-CNT interface implying the predominant growth mechanism is root growth. There are a few instances where the catalyst particle moved several nanometers into the CNT length, away from the interface. Growth patterns similar to this indicate a small probability of catalyst lift-off.

Figure 4 shows an energy-filtered transmission electron microscope (EF-TEM) image of an MPE-CVD film. It can be seen that iron particles in this case do not reside along the interface, but rather move easily into the CNT, away from the interface. The mobility of nonanchored catalytic particles at the interface may also contribute to disordered CNT growth.

As seen in Figure 5, EF-TEM analysis of the FCT-CVD sample indicates a high distribution of iron particles residing at the diamond CNT interface which has the silica functional layer. There are additional iron particles found further away in the CNT forest. This is expected due to the continuous iron particle introduction throughout the CNT growth phase. The large number of iron catalysts at the interface suggests that the silica nanolayer keeps them anchored and prevents them from migrating during CNT growth.

Figure 6 shows the average particle size distribution taken with Image J software. This software provides qualitative values for the catalyst particle size and tube diameter comparison taken from the resulting TEM images. For T-CVD, the 
Scan carbon

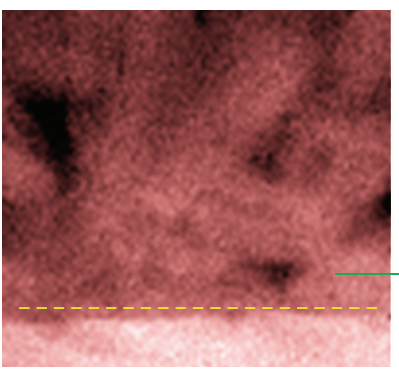

Scan area

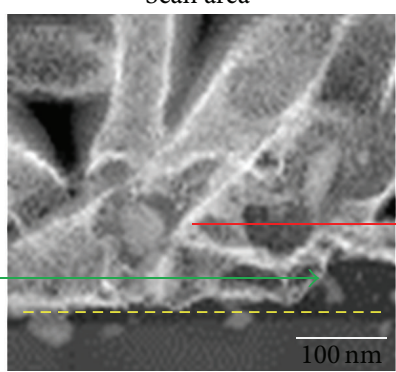

Scan nickel catalyst

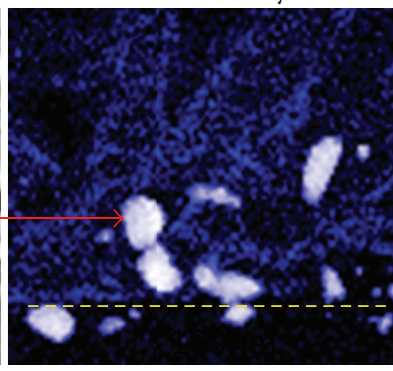

FIgure 3: Cross-sectional TEM image of T-CVD-grown CNTs, the scale bar is $100 \mathrm{~nm}$.

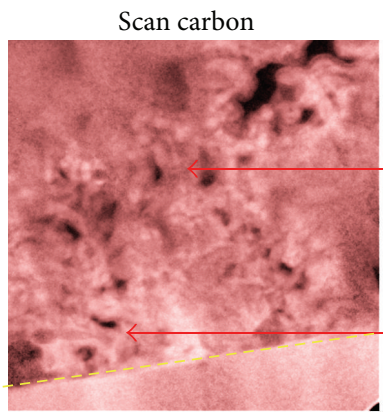

Scan area

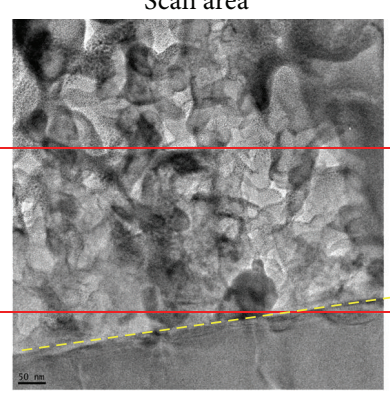

Scan iron catalyst

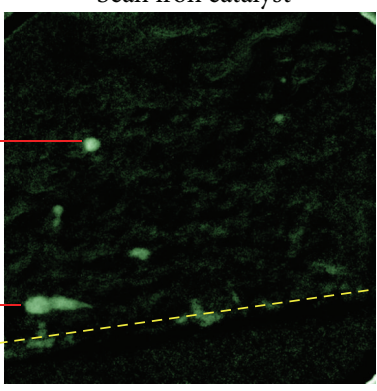

FIgure 4: Cross-sectional TEM image of MPE-CVD-grown CNTs, the scale bar is $50 \mathrm{~nm}$.

average catalyst particle diameter was measured to be $53 \mathrm{~nm} \pm$ $16 \mathrm{~nm}$, and the average tube diameter was $61 \mathrm{~nm} \pm 12 \mathrm{~nm}$. Thus, the catalyst diameters measured using TEM images are within the margin of error with the top view images seen by SEM. For MPE-CVD, the average catalyst particle diameter was $21 \mathrm{~nm} \pm 7 \mathrm{~nm}$, and the average tube diameter was $40 \mathrm{~nm} \pm 18 \mathrm{~nm}$. For the FCT-CVD method, the average catalyst particle size was $12 \mathrm{~nm} \pm 2 \mathrm{~nm}$, and the average tube diameter was $12 \mathrm{~nm} \pm 2 \mathrm{~nm}$. Cross-sectional TEM images provided additional information about particle location and concentration relative to the interface. For T-CVD, the image indicated that catalyst particles are the largest of all growth methods and reside close to the interface. For MPE-CVD, the catalyst particles are distributed away from the interface. This migration may be due to either the fact that they are smaller in size compared to T-CVD samples, the excess energy provided by the hydrogen plasma, or both. For FCT-CVD, the image indicates that the majority of the iron particles used as catalysts are anchored along the $\mathrm{SiO}_{2}$ layer and the iron particles that arrived later reside outside the growing CNTs. The anchoring of smaller nanoparticles may be caused by the silica functional layer and may assist in uniform CNT growth. It can be seen that the nanocatalyst particle size in each case correlates with the CNT diameters observed by SEM and TEM and further validates the hypothesis that the CNT size is largely governed by catalyst particles.

3.4. Raman Spectrograph Results. Raman spectroscopy was performed on samples produced by the different growth methods. The results are presented in Figure 7. According to the Handbook of Raman Spectroscopy and Dresselhaus et al., Raman spectroscopy can be used as a guide to describe different carbon structures, such as diamond-like carbon which has $\mathrm{C}-\mathrm{C} \mathrm{sp}^{3}$ bonding, graphitic carbon which has $\mathrm{sp}^{2}$ bonding, and glassy/amorphous carbon which has $\mathrm{C}-\mathrm{H}$ and disordered mixed bonds $[25,30,31]$. The peaks found around $1350-1365 \mathrm{~cm}^{-1}$ are called $D$-peaks, resulting from the disorderly network of $\mathrm{sp}^{2}$ and $\mathrm{sp}^{3}$ carbon clusters, whereas the peaks found around $1580-1620 \mathrm{~cm}^{-1}$ are called $G$-peaks as a result of graphite, and finally the peak found at $1332 \mathrm{~cm}^{-1}$ is the diamond peak [30-32]. The most interesting finding from our experiment concerned the calculated intensity of the $D$-peak to $G$-peak ratios $\left(I_{D} / I_{G}\right)$. This ratio matched the CNT morphology in terms of defect appearance in the SEM. The $I_{D} / I_{G}$ ratios are given as follows: T-CVD $=0.30$; MPECVD $=1.94$; FCT-CVD $=0.84$. Note that the T-CVD has the lowest $I_{D} / I_{G}$ ratio; its SEM images indicated that this tube structure looks the smoothest. In contrast, the $I_{D} / I_{G}$ for MPE-CVD is the highest and its SEM images indicated that the tube structure appears damaged and full of defects. Earlier, we stated that hydrogen may etch the CNT and introduce defect sites that can raise the intensity of the $D$ peak. Another possibility that causes high $D$-peak is when $\mathrm{CNT}$ is introduced to a hydrogen rich environment; the hydrogen alters CNT's C-H bonding and transforms them into diamond or disordered carbon $[27,33]$. If this is the case, it explains why the disordered bonding peak, the $D$-peak, is much higher in the MPE-CVD sample.

Since the CNTs were grown on diamond substrates, one might suggest that the high intensity of $D$-peak resulted from signals coming from the diamond substrate underneath. However, the recorded $D$-peak intensity lies around 

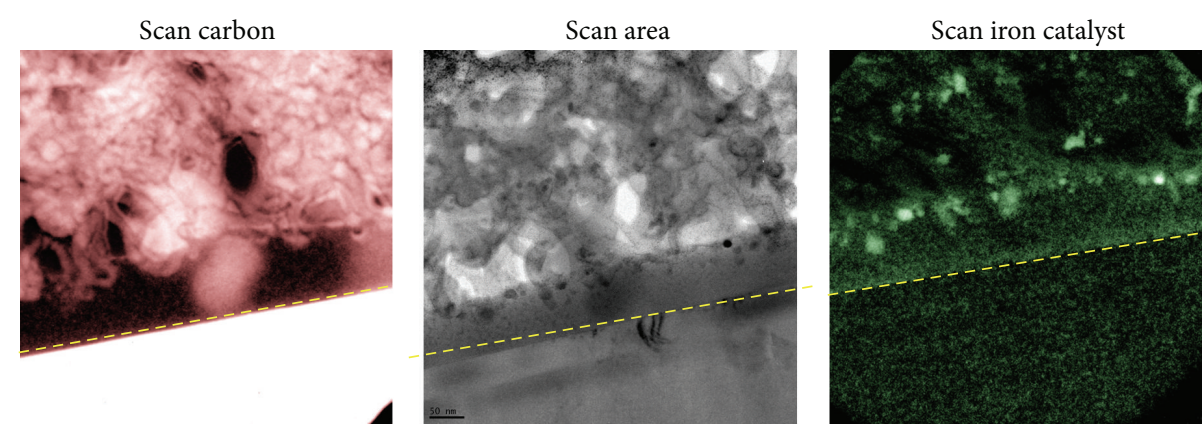

Figure 5: Cross-sectional TEM image of FCT-CVD-grown CNTs, the scale bar is $50 \mathrm{~nm}$.

Average catalytic particle size and CNTs diameter distribution

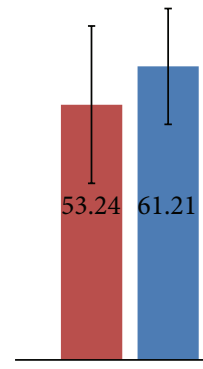

T-CVD

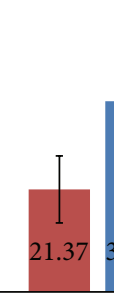

MPE-CVD
12.1811 .61

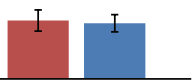

FCT-CVD
- Average catalytic particle size

- Average CNTs diameter

FIGURE 6: Particle size and CNTs tube diameter distribution chart.

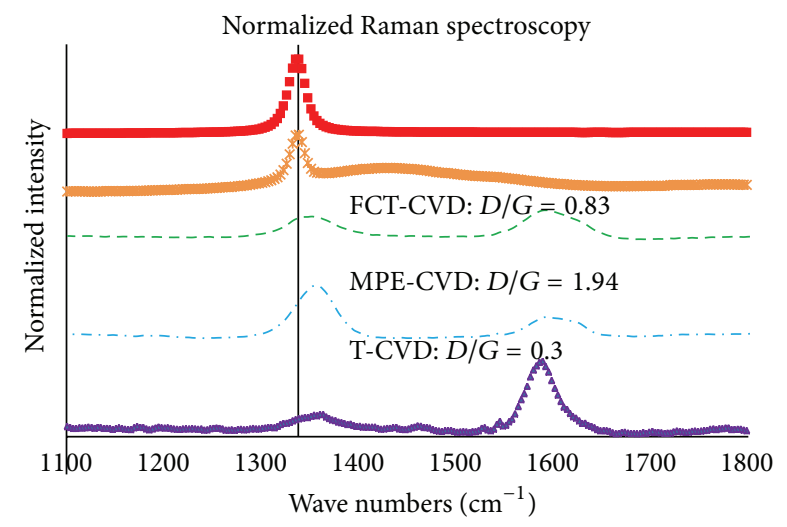

$$
\begin{array}{ll}
\text { - Natural diamond } & -- \text { MPE-CVD } \\
* & \text { Synthetic diamond } \\
--- & \text { FCT-CVD }
\end{array}
$$

FIGURE 7: Raman spectroscopy signals for natural diamond, synthetic diamond, and CNTs from the three CVD methods.

$\left(1354 \mathrm{~cm}^{-1}\right)$, whereas known diamond $D$-peaks are detected at $\left(1332 \mathrm{~cm}^{-1}\right)$, indicating that the peak signal did not result from the diamond substrate. For confirmation, a razor blade was used to scrape away the CNTs from a sample and expose the diamond substrate underneath. The Raman spectra taken from the exposed diamond surface has a peak intensity at $\left(1338 \mathrm{~cm}^{-1}\right)$, which closely matches the published Raman peak for diamond $\left(1332 \mathrm{~cm}^{-1}\right)$. This additional test further indicates that the $\left(1354 \mathrm{~cm}^{-1}\right)$ peak is indeed the $D$-peak from the disordered CNT and not from the diamond substrate.

3.5. Sonication Results. If the sample was used in a commercial device, it would be important to know if the CNTs had the strength to stay intact within the substrate. The sonication test was used as a qualitative comparison of the forces needed to detach CNT from the substrate [34-36]. SEM images were taken before and after the sonication tests as shown in Figure 8. The images in the top row of Figure 8 were taken prior to sonication, and the bottom row images were taken after the test. Figures 8(a) were form the T-CVD, Figures 8(b) were from the MPE-CVD, Figures 8(c) were from FCT-CVD, and Figures 8(d) were optical images taken from MPE-CVD sample. The before sonication image seen in Figure 8 (d) can also be used to represent T-CVD and FCT-CVD, because there were no visual differences observed after the sonication test. It can be seen that the nanotubes were intact even after sonication and that there were no noticeable differences seen in the before and after images from SEM. The low-magnification optical images show a difference for the MPE-CVD sample. Before sonication, all samples look identical at this magnification as seen in Figure 8(d) top image. The optical images after the sonication test were unchanged for T-CVD and FCT-CVD which are also represented by Figure $8(\mathrm{~d})$ top image. The MPE-CVD after sonication sample was different as shown in Figure 8(d) bottom image. While postsonication SEM images of MPE-CVD sample show that CNTs were present on the scanned regions, the low-magnification optical images show exposed diamond substrate on the corners of the sample. This indicates detachment of CNTs at the corners of this sample, implying that the bonding between CNTs and substrate may be the weakest in this fabrication method. It must be noted that because of the extremely high length/diameter ratio of the nanotubes, agitation in an ultrasonic bath produces concentrated stresses at the root of the CNT. Survival under these conditions indicates that these materials will be robust in many service conditions. Among the three samples, MPECVD specimen may be the weakest, and detachment begins 

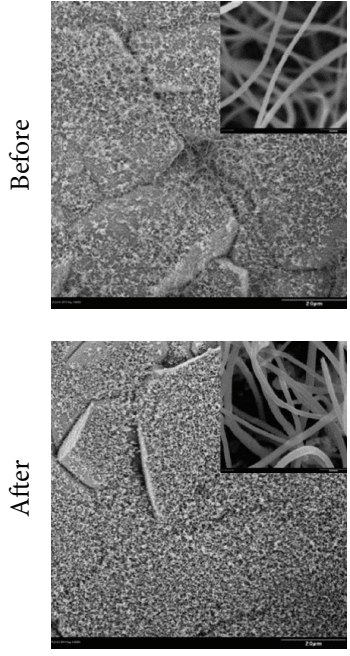

(a)


(b)
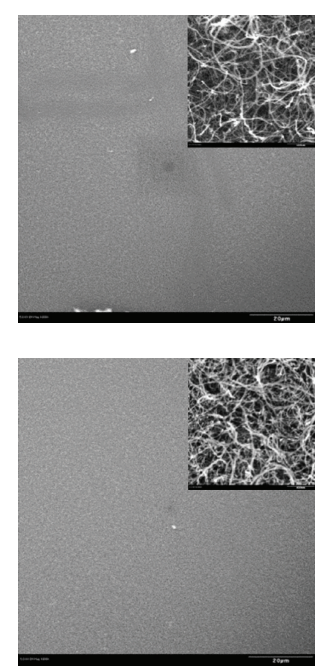

(c)
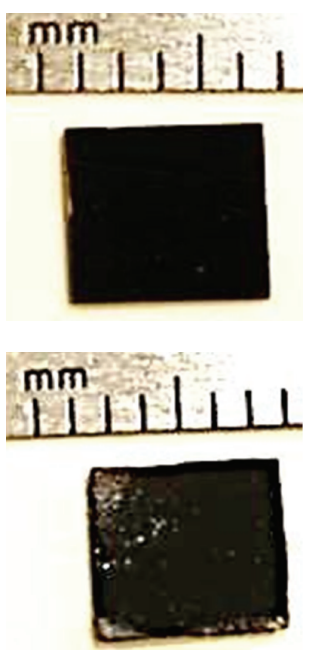

(d)

FIGURE 8: (a), (b), and (c) are SEM images, and (d) is the optical image. The images in the top row were taken prior to sonication, and those in the bottom row were taken after the test. (a) is the T-CVD sample. (b) is the MPE-CVD sample. (c) is the FCT-CVD sample, and (d) is taken from MPE-CVD Sample. There were no visual changes seen after the sonication test for the T-CVD and the FCT-CVD sample. Therefore, the before image from (d), can also be used to represent before and after sonication test for T-CVD and FCT-CVD sample.

to occur at the corners, which are the most vulnerable regions of the sample.

3.6. Overall Comparison. It is seen that very different types of CNT layers can be grown on diamond film by different techniques. It must be noted that T-CVD and MPE-CVD are multistep processes that separate the catalyst deposition step from the CNT growth step. The catalytic particles in the TCVD growth are found to be the largest of the three types of growth for the conditions used. These particles were found to reside at the interface with minimal migration into the CNT layer. The resulting CNT structures were found to be the smoothest with the largest tube diameters of all three growth types. This smooth structure may be useful for electronic and thermal applications that require minimal defects.

The SEM images show the CNTs that resulted from the MPE-CVD method contains kinks and defects on the individual tubes. The defects may be the result of hydrogen plasma and excess hydrogen in the chamber. The excess hydrogen can etch the surface of CNTs as it grows, which results in a defected surface. The hydrogen plasma may also provide excess energy that inadvertently promotes catalyst migration. If this migration occurs during CNT growth, it may be another underlying reason as to why those CNT structures are kinked. It can be proposed that as the CNTs are growing with the migrating catalytic particles, a straight path for growth was not provided, which resulted in a kinkedtype growth. Therefore, this approach which is suitable for large area growth will be useful only in situations where defective and kinked CNTs are acceptable. It would be interesting to determine if the defective CNTs grown under energetic conditions are more reactive than their smoother counterparts. Another noticeable difference provided by the MPE-CVD method is that it has the weakest bonding with the substrate compared to the other two methods. Images from the three growth methods were compared after the sonication test. The images from the sonication test show the corners of the diamond substrate were exposed after the test, which is not seen in the other samples. The benefit of using FCTCVD is that it is a one step process where catalyst and carbon source are introduced together. The resulting CNTs grown by this process had the smallest diameter, averaging $12 \mathrm{~nm}$, with uniform and dense growth patterns. While this may be a very suitable approach in many applications, the disadvantage is the possibility of excess iron particles arising from continuous catalyst deposition. This may be mitigated by modifying the process to cut off ferrocene during the latter part of CNT growth.

Since each method has its unique advantages and disadvantages, these studies indicate that the selection of the CNT growth method is heavily influenced by the intended application.

\section{Conclusions}

In this paper, we have compared the growth of CNTs forests on synthetic diamond substrates using three different CVD growth methods. The CNTs were characterized using electron microscope imaging (SEM and TEM), elemental analysis (EDS), Raman spectroscopy, and the sonication test. The SEM and TEM images indicated each method produces CNTs with a distinctly different diameter and morphology. Raman spectroscopic analysis suggested that T-CVD had the lowest disordered carbon to graphite intensity ratio of 0.3 followed by FCT-CVD of 0.83 and the highest by MPECVD of 1.94. T-CVD also produces large diameter CNTs that are otherwise clean, with minimal tubular defects or additional catalytic contaminants. MPE-CVD is sometimes 
the preferred method for larger scaled applications; however, the excess hydrogen plasma energy during growth may cause migrations of catalytic particles, surface etching, and defective CNTs that may be detrimental to precision applications. MPE-CVD has the highest probability for catalytic mobility, the most defective CNTs structure morphology, and the weakest interfacial bonding strength. It may be possible to improve this situation through extensive processing parameter changes, but not certain. FCT-CVD has the advantage of being a one-step CNT growth method that does not need a separate catalyst deposition step, yet produces dense uniform CNTs. This technique provided the finest CNT diameter, and intermediate diamond/graphite ratio in the Raman signal. This method does produce some excess metal particles due to continuous catalyst nucleation. However, this issue can be easily addressed in the future if needed, by stopping the catalytic source after a certain growth time. Finally, this study indicates that there is a clear correlation between the size of catalyst particle and the CNT diameters.

\section{Conflict of Interests}

B. Quinton, as the author of this paper, dose not have a direct financial relation with the incorporation and software mentioned in this paper and that might lead to a conflict of interests for any of the authors.

\section{Acknowledgments}

The authors of the paper would like to thank the funding support of Air Force Office of Scientific Research, Air Force Research Laboratory Propulsion Directorate, Wright State University, and Ohio Board of Regents. The authors would like to acknowledge Dr. Robert Wheeler for his guidance on TEM and EDS.

\section{References}

[1] M. Meyyappan, Carbon Nanotubes: Science and Applications, CRC Press, Boca Raton, Fla, USA, 2004.

[2] S. Iijima, "Helical microtubules of graphitic carbon," Nature, vol. 354, no. 6348, pp. 56-58, 1991.

[3] R. Saito, M. Fujita, G. Dresselhaus, and M. S. Dresselhaus, "Electronic structure of chiral graphene tubules," Applied Physics Letters, vol. 60, no. 18, pp. 2204-2206, 1992.

[4] P. Calvert, "Strength in disunity," Nature, vol. 357, no. 6377, pp. 365-366, 1992.

[5] N. V. Novikov and S. N. Dub, "Hardness and fracture toughness of CVD diamond film," Diamond and Related Materials, vol. 5, no. 9, pp. 1026-1030, 1996.

[6] S. V. Kidalov and F. M. Shakhov, "Thermal conductivity of diamond composites," Materials, vol. 2, pp. 2467-2495, 2009.

[7] C. J. Lee, S. C. Lyu, Y. R. Cho, J. H. Lee, and K. I. Cho, “Diametercontrolled growth of carbon nanotubes using thermal chemical vapor deposition," Chemical Physics Letters, vol. 341, no. 3-4, pp. 245-249, 2001.

[8] E. T. Thostenson, Z. Ren, and T. W. Chou, "Advances in the science and technology of carbon nanotubes and their composites: a review," Composites Science and Technology, vol. 61, no. 13, pp. 1899-1912, 2001.

[9] N. Tumilty, L. Kasharina, T. Prokhoda, B. Sinelnikov, and R. B. Jackman, "Synthesis of carbon nanotubes on single crystal diamond," Carbon, vol. 48, no. 11, pp. 3027-3032, 2010.

[10] C. Varanasi, J. Petry, L. Brunke et al., "Growth of high-quality carbon nanotubes on free-standing diamond substrates," Carbon, vol. 48, no. 9, pp. 2442-2446, 2010.

[11] D. Takagi, Y. Kobayashi, and Y. Homma, "Carbon nanotube growth from diamond," Journal of the American Chemical Society, vol. 131, no. 20, pp. 6922-6923, 2009.

[12] Y. Li, W. Kim, Y. Zhang, M. Rolandi, D. Wang, and H. Dai, "Growth of single-walled carbon nanotubes from discrete catalytic nanoparticles of various sizes," Journal of Physical Chemistry B, vol. 105, no. 46, pp. 11424-11431, 2001.

[13] S. Helveg, C. López-Cartes, J. Sehested et al., "Atomic-scale imaging of carbon nanofibre growth," Nature, vol. 427, no. 6973, pp. 426-429, 2004.

[14] R. T. K. Baker, M. A. Barber, P. S. Harris, F. S. Feates, and R. J. Waite, "Nucleation and growth of carbon deposits from the nickel catalyzed decomposition of acetylene," Journal of Catalysis, vol. 26, no. 1, pp. 51-62, 1972.

[15] C. T. Wirth, S. Hofmann, and J. Robertson, "State of the catalyst during carbon nanotube growth," Diamond and Related Materials, vol. 18, pp. 940-945, 2009.

[16] J. Y. Raty, F. Gygi, and G. Galli, "Growth of carbon nanotubes on metal nanoparticles: a microscopic mechanism from ab initio molecular dynamics simulations," Physical Review Letters, vol. 95, no. 9, Article ID 096103, 4 pages, 2005.

[17] S. Hofmann, G. Csányi, A. Ferrari, M. Payne, and J. Robertson, "Surface diffusion: the low activation energy path for nanotube growth," Physical Review Letters, vol. 95, Article ID 036101, 4 pages, 2005.

[18] K. Koziol, B. O. Boskovic, and N. Yahya, Carbon and Oxide Nanostructures: Synthesis, Characterisation and Applications, Henry Dickens, Columbia, SC, USA, 2011.

[19] Y. C. Choi, D. J. Bae, Y. H. Lee et al., "Growth of carbon nanotubes by microwave plasma-enhanced chemical vapor deposition at low temperature," Journal of Vacuum Science and Technology A, vol. 18, no. 4, pp. 1864-1868, 2000.

[20] Teo, B. K. Kenneth, C. Singh, M. Chhowalla, and W. Milne, Encyclopedia of Nanoscience and Nanotechnology, 2003.

[21] A. J. S. Fernandes, M. Pinto, M. A. Neto, F. J. Oliveira, R. F. Silva, and F. M. Costa, "Nano carbon hybrids from the simultaneous synthesis of CNT/NCD by MPCVD," Diamond and Related Materials, vol. 18, no. 2-3, pp. 160-163, 2009.

[22] A. Cao, P. M. Ajayan, G. Ramanath, R. Baskaran, and K. Turner, "Silicon oxide thickness-dependent growth of carbon nanotubes," Applied Physics Letters, vol. 84, no. 1, pp. 109-111, 2004.

[23] S. M. Mukhopadhyay, A. Karumuri, and I. T. Barney, "Hierarchical nanostructures by nanotube grafting on porous cellular surfaces," Journal of Physics D, vol. 42, no. 19, Article ID 195503, 2009.

[24] S. M. Mukhopadhyay, P. Joshi, and R. V. Pulikollu, “Thin films for coating nanomaterials," Tsinghua Science \& Technology, vol. 10, no. 6, pp. 709-717, 2005.

[25] J. L. Lauer, Handbook of Raman Spectroscopy from the Research Laboratory to the Process Line, Marcel Dekker, West Yorkshire, UK, 1st edition, 2001. 
[26] Y. Homma, Y. Kobayashi, T. Ogino et al., "Role of transition metal catalysts in single-walled carbon nanotube growth in chemical vapor deposition," Journal of Physical Chemistry B, vol. 107, no. 44, pp. 12161-12164, 2003.

[27] M. J. Behr, E. A. Gaulding, K. A. Mkhoyan, and E. S. Aydil, "Hydrogen etching and cutting of multiwall carbon nanotubes," Journal of Vacuum Science \& Technology B, vol. 28, no. 6, pp. 1187-1194, 2010.

[28] L. T. Sun, J. L. Gong, Z. Y. Zhu et al., "Nanocrystalline diamond from carbon nanotubes," Applied Physics Letters, vol. 84, no. 15, pp. 2901-2903, 2004.

[29] Q. Yang, S. Yang, C. Xiao, and A. Hirose, “Transformation of carbon nanotubes to diamond in microwave hydrogen plasma," Materials Letters, vol. 61, no. 11-12, pp. 2208-2211, 2007.

[30] M. S. Dresselhaus, G. Dresselhaus, R. Saito, and A. Jorio, "Raman spectroscopy of carbon nanotubes," Physics Reports, vol. 409, no. 2, pp. 47-99, 2005.

[31] M. S. Dresselhaus, A. Jorio, A. G. Souza Filho, and R. Saito, "Defect characterization in graphene and carbon nanotubes using Raman spectroscopy," Philosophical Transactions of the Royal Society A, vol. 368, no. 1932, pp. 5355-5377, 2010.

[32] L. C. O’brien, R. L. Kubicek, and J. J. O’brien, "Laser raman spectroscopy of diamond," Journal of Chemical Education, vol. 71, no. 9, pp. 759-760, 1994.

[33] G. Zhang, P. Qi, X. Wang et al., "Hydrogenation and hydrocarbonation and etching of single-walled carbon nanotubes," Journal of the American Chemical Society, vol. 128, no. 18, pp. 6026-6027, 2006.

[34] Q. Zhang, J. Liu, R. Sager, L. Dai, and J. Baur, "Hierarchical composites of carbon nanotubes on carbon fiber: influence of growth condition on fiber tensile properties," Composites Science and Technology, vol. 69, no. 5, pp. 594-601, 2009.

[35] M. F. De Riccardis, D. Carbone, T. D. Makris, R. Giorgi, N. Lisi, and E. Salernitano, "Anchorage of carbon nanotubes grown on carbon fibres," Carbon, vol. 44, no. 4, pp. 671-674, 2006.

[36] I. T. Barney, Fabrication and Testing of Hierarchical Carbon Nanostructures For Multifunctional Applications, Wright State University, 2012. 

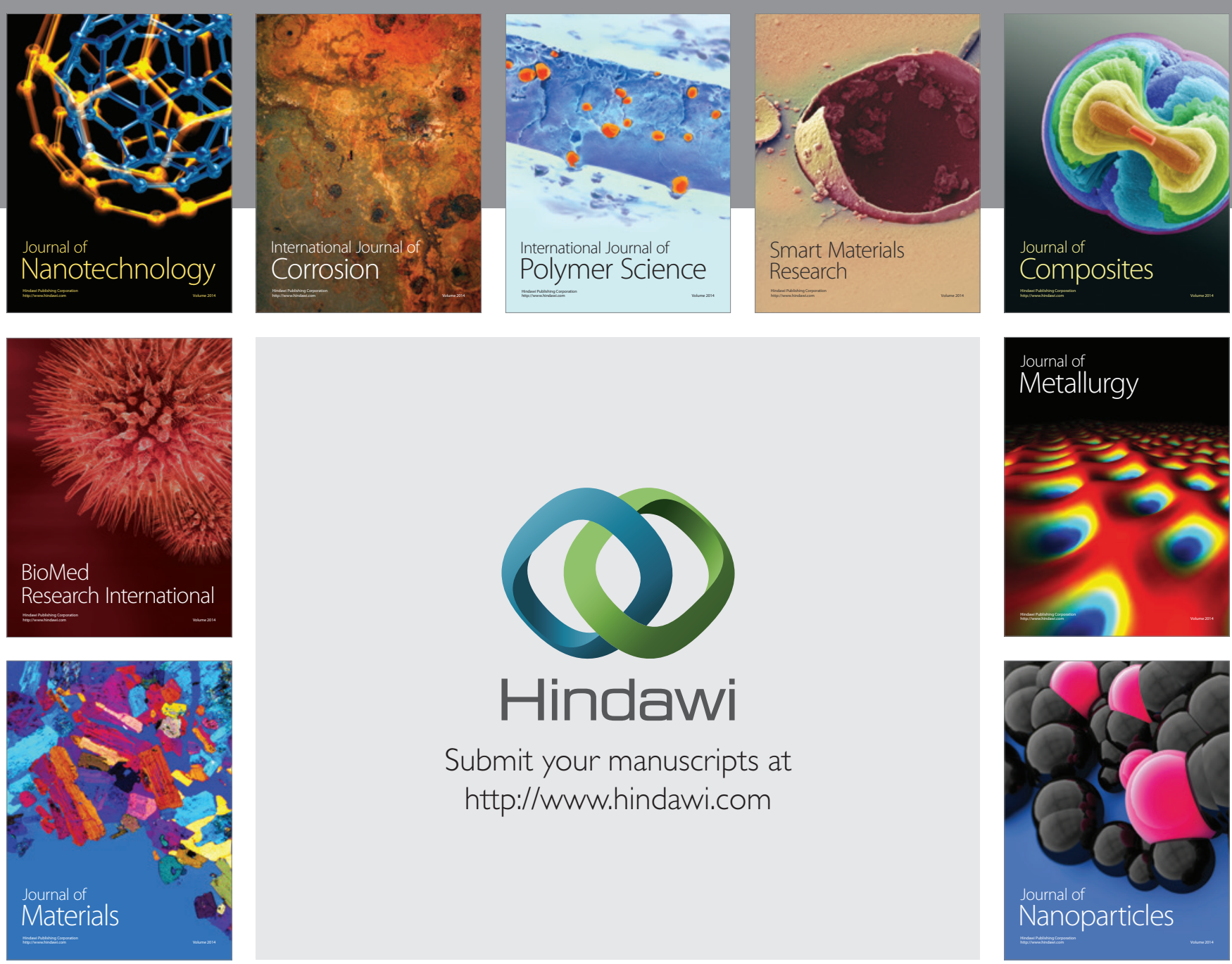

Submit your manuscripts at http://www.hindawi.com
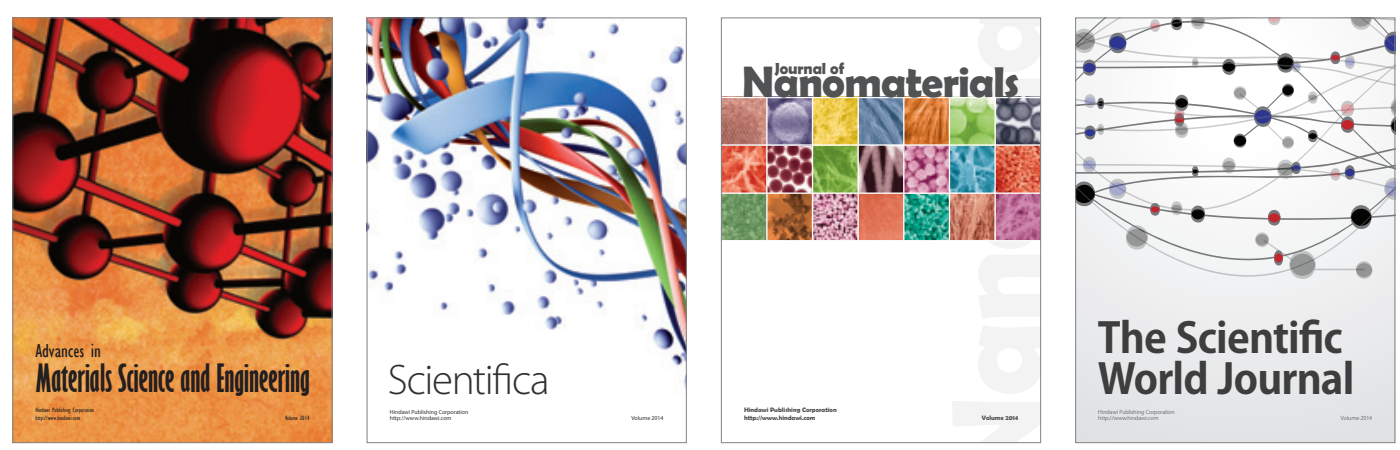

\section{The Scientific World Journal}
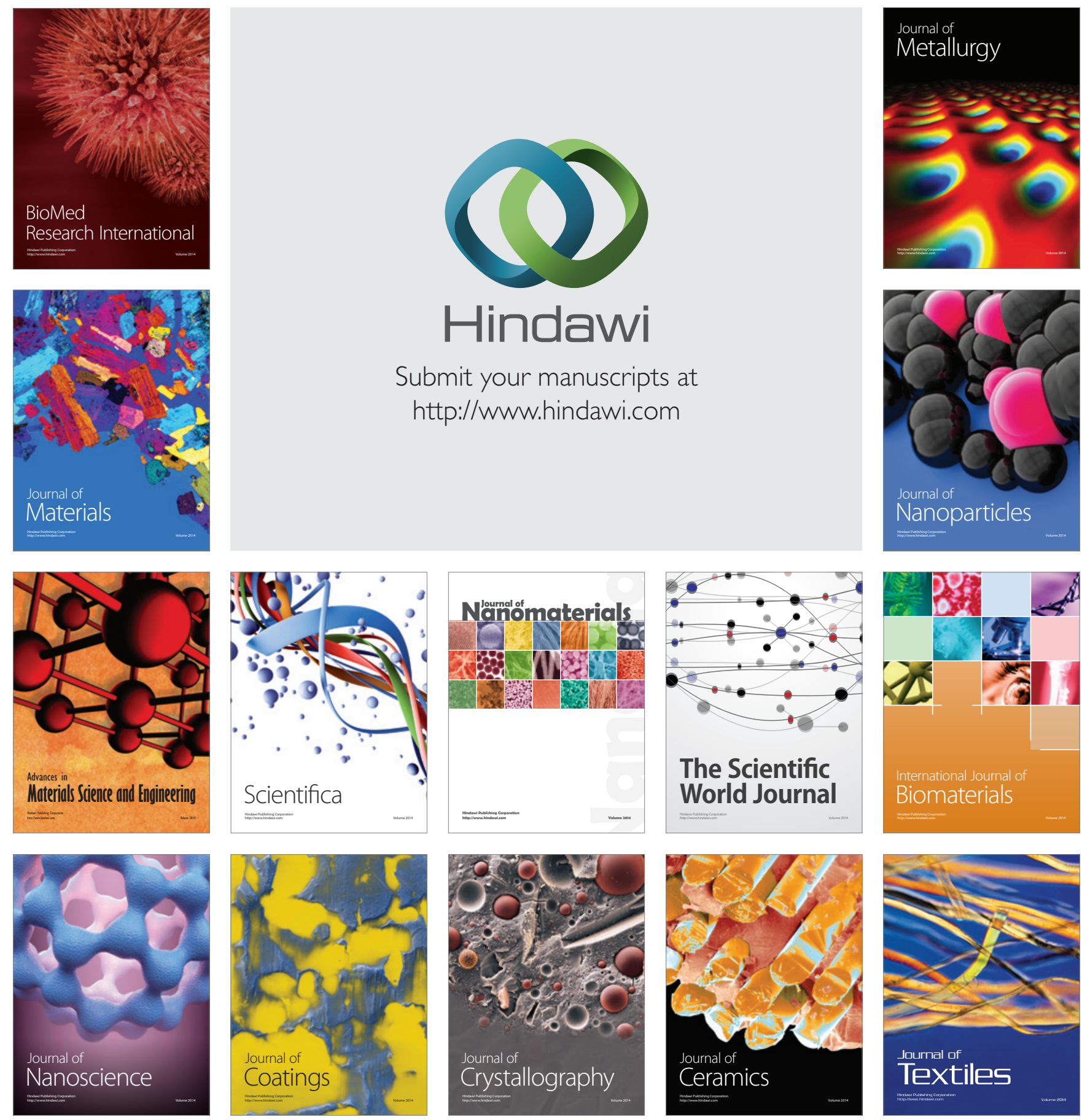